\title{
Improved Finite Time in Eliminating Disagreement of Opinion Dynamics via Noise
}

\author{
Yong Ding ${ }^{1}$ and Lipo Mo ${ }^{2}$ \\ ${ }^{1}$ School of General Education, Weifang University of Science and Technology, Shouguang 262700, China \\ ${ }^{2}$ School of Science, Beijing Technology and Business University, Beijing 100048, China \\ Correspondence should be addressed to Lipo Mo; beihangmlp@126.com
}

Received 25 May 2017; Revised 7 September 2017; Accepted 27 September 2017; Published 19 October 2017

Academic Editor: Antonio Scarfone

Copyright (c) 2017 Yong Ding and Lipo Mo. This is an open access article distributed under the Creative Commons Attribution License, which permits unrestricted use, distribution, and reproduction in any medium, provided the original work is properly cited.

\begin{abstract}
We aim to refine the estimation of the finite stopping time when the disagreement in an opinion group is eliminated by a simple but novel noise intervened strategy. It has been proved that, by using this noise intervened control strategy, the divisive opinions would get synchronized in finite time. Moreover, the finite stopping time when resolving the disagreement has been clarified. The estimation of the finite stopping time will effectively reveal which factors and how they determine the consequence of intervention. However, the upper bound for the estimation of the integrable stopping time when noise is oriented has been quite conservative. In this paper, we investigate the finite stopping time of eliminating the disagreement by completely oriented noise and a much more precise formula for the estimation of the finite stopping time is obtained finally via direct calculation.
\end{abstract}

\section{Introduction}

In the recent decades, modeling and analysis of opinion dynamics are becoming a much attractive area. More and more researchers from various fields have paid attention to it $[1,2]$, and several agent-based mathematical models have been established to investigate the evolution of opinion dynamics [3-13]. In these models, the evolution of the opinions can be determined by the interaction topology of agents which are based on the preassumed graph or the bounded confidence (BC) of the agents. Using these models, the complex evolution of opinion dynamics was effectively studied and plenty of opinion behaviors were revealed, such as the basic agreement or disagreement of the opinions and the final structure of the opinion system.

One of the emerging fields in studying the opinion dynamics is how the noise influences the evolution of the social opinions. It is known that the noise is ubiquitous in opinion dynamics and intervenes during the evolution of one's opinion from a wide range of aspects, such as information flow from free media of broadcast, newspaper, TV, and so on, the contaminated transmission channels, or even one's free will. The investigations were first carried out by some simulation methods in [14-17], and with both continuous and discrete opinion models some interesting phenomena were discovered, among which a focus is that the noise in some situations could play a positive role in enhancing the consensus of opinions. Actually, the similar positive role noise plays has been found in various areas in the past decades [1822]. In opinion dynamics, the complete theoretical analysis of a continuous noisy opinion dynamics was first established in [23] very recently. In [23], based on the well-known Hegselmann-Krause (HK) confidence model, it was strictly proved that the noise could almost surely induce the opinions to achieve quasi-consensus in finite time, and also the critical noise strength is obtained. These results not only reveal the richer mechanisms of the opinion evolution, but also provide new insights into designing noise intervention strategy to eliminate the disagreement in a group.

Soon after, inspired by the achievements in [23], a very simple but practical noise intervention strategy was designed in [24]. In their scheme, very weak noises uniformly distributed on $\left[-\delta_{1}, \delta_{2}\right]\left(0<\delta_{1} \leq \delta_{2}\right)$ were persistently introduced to only one agent in a divisive group described by HK dynamics, and it was strictly proved that the disagreement in the group was eliminated in finite time. In 
particular, the finite time when the disagreement got resolved was firstly estimated in this paper. The time is actually a finite stopping time, and its expectation was shown to be infinite when the noise is neutral (i.e., $\delta_{1}=\delta_{2}$ ). When the noise is oriented, that is, $\delta_{1}<\delta_{2}$, the expectation of the stopping time was shown to be finite and a upper bound was obtained. The formula of the estimation of the integrable stopping time when the noise is oriented embeds all the factors that could determine the effectiveness of noise intervention. In this sense, calculating the stopping time possesses its own significance in investigating the design of noise intervention strategy. However, the estimation of the upper bound is quite a bit conservative in [23]. Consequently, a natural concern in the following is whether this conservative upper bound can be modified, at least for some special noise case.

In this paper, we will follow the path in [24] and further directly calculate the stopping time when the disagreement is eliminated under the case of completely oriented noise, that is, $\delta_{1}=0, \delta_{2}>0$, where indeed a much more precise formula is obtained. To be specific, while the expectation of finite stopping time under completely oriented noise is $(2 n / \delta)\left(x_{2}^{*}-x_{1}^{*}\right)+2 n$ using the formula obtained in [24], it is refined in this paper to be $(e n / 2 \delta)\left(x_{2}^{*}-x_{1}^{*}\right)-(e \epsilon / 2 \delta) n+2+e$, where $n$ is the size of the group and the other parameters are fixed and nonnegative with $x_{2}^{*}-x_{1}^{*}>\epsilon$. Since an at least decrease of $(2+\epsilon / \delta) n$ is obtained, the stopping time is much refined here, especially when the group size is large.

The rest of the paper is organized as follows: Section 2 presents the model and the formulation, Section 3 gives the main results of the paper, and Section 4 shows some simulations, while some concluding remarks are given in Section 5 .

\section{Basic Model and Preliminaries}

In this part, we will introduce the basic divisive opinion model in line with that in [24]. It is known that the disagreement phenomenon of opinion dynamics can be well characterized by the HK dynamics:

$$
x_{i}(t+1)=\frac{1}{\left|\mathcal{N}_{i}(x(t))\right|} \sum_{j \in \mathcal{N}_{i}(x(t))} x_{j}(t), \quad i \in \mathscr{V},
$$

where $n$ is the size of the group with $\mathscr{V}=\{1, \ldots, n\}$ and $x(t)$ is opinion values at time $t$ which takes values in $(-\infty, \infty)^{n}$. Further,

$$
\mathcal{N}_{i}(x(t))=\left\{j \in \mathscr{V}|| x_{j}(t)-x_{i}(t) \mid \leq \epsilon\right\}
$$

is the neighbor set of $i$ and $\epsilon>0$ represents the confidence threshold of the agents. Here, $|\cdot|$ can be the cardinal number of a set or the absolute value of a real number accordingly.

The noise-free HK model (1) has proved that the opinion will reach static state in finite time, with agreement or disagreement status.

Proposition 1 (see [9]). For every $1 \leq i \leq n$ of (1), $x_{i}(t)$ will converge to some $x_{i}^{*}$ in finite time, and either $x_{i}^{*}=x_{j}^{*}$ or $\mid x_{i}^{*}-$ $x_{j}^{*} \mid>\epsilon$ holds for any $i, j$.
If $x_{i}^{*}=x_{j}^{*}$ for all $i, j$, the opinions reach consensus and the group realizes agreement, or the opinions get separated and the disagreement occurs otherwise.

To describe the divisive opinion system, $N_{c}$ distinct standpoints $x_{i}^{*}\left(1 \leq i \leq N_{c}\right)$ are assumed in the divisive system in [24]. Here, for simplicity, we suppose $N_{c}=2$ and the bidivisive system can be described as

$$
\begin{aligned}
\mathscr{V} & =\mathscr{V}_{1} \bigcup \mathscr{V}_{2}, \quad \mathscr{V}_{1} \bigcap \mathscr{V}_{2}=\varnothing, \\
x_{i}(0) & =x_{g}^{*} \in(-\infty, \infty), \quad \text { for } i \in \mathscr{V}_{g}, 1 \leq g \leq 2, \\
x_{2}^{*}-x_{1}^{*} & >\epsilon .
\end{aligned}
$$

Following the intervention scheme in [24] where noise intervention is added to agent 1 , the noise intervention model of (1)-(3) here is also, for $i \in \mathscr{V}$,

$$
x_{i}(t+1)=\frac{1}{\left|\mathcal{N}_{i}(x(t))\right|} \sum_{j \in \mathcal{N}_{i}(x(t))} x_{j}(t)+I_{\{i=1\}} \xi(t+1),
$$

where the noises $\{\xi(t), t \geq 1\}$ are independent and uniformly distributed on a completely oriented interval

$$
[0, \delta], \quad \delta>0
$$

and $I_{\{\cdot\}}$ is the indicator function with $I_{\{i=1\}}=1$ when $i=1$ and $I_{\{i=1\}}=0$ otherwise.

Moreover, to describe the behavior of model (2)-(5) conveniently, a definition of consensus in the noisy case is introduced.

Definition 2. Define

$$
\begin{aligned}
d_{\mathscr{V}}(t) & =\max _{i, j \in \mathscr{V}}\left|x_{i}(t)-x_{j}(t)\right|, \\
d_{\mathscr{V}} & =\limsup _{t \rightarrow \infty} d_{\mathscr{V}}(t) .
\end{aligned}
$$

For $\phi \in[0, \infty)$,

(i) if $d_{\mathscr{V}} \leq \phi$, we say the system (2)-(5) will reach $\phi$ consensus;

(ii) if $P\left\{d_{\mathscr{V}} \leq \phi\right\}=1$, we say almost surely (a.s.) that system (2)-(5) will reach $\phi$-consensus;

(iii) let $T=\inf \left\{t: d_{\mathscr{V}}\left(t^{\prime}\right) \leq \phi\right.$ for all $\left.t^{\prime} \geq t\right\}$; if $P\{T<$ $\infty\}=1$, we say a.s. that system (2)-(5) reaches $\phi$-consensus in finite time.

With the above preliminaries, the $\delta$-consensus of system (2)-(5) and the estimation of the finite stopping time were obtained in [24].

Proposition 3. Define $T=\inf \left\{t: d_{\mathscr{V}}(t) \leq \epsilon\right\}$ and let $\delta \leq \epsilon /$ $2 n$; then $P\{T<\infty\}=1$ and the system will reach $\delta$-consensus at $T+1$. Moreover,

$$
\mathbf{E} T \leq \frac{2 n}{\delta}\left(x_{2}^{*}-x_{1}^{*}+\delta\right) .
$$

It can be found from (7) that the upper bound of the stopping time is determined by the group size $n$, the noise strength $\delta$, and the initial opinion difference $x_{2}^{*}-x_{1}^{*}$. Though (7) gives a quite concise estimation for the stopping time $T$, the main results displayed in the next section will show that this upper bound can be refined largely. 


\section{Main Results}

To be straightforward, we first give the main result.

Theorem 4. Let $T=\inf \left\{t: d_{\mathscr{V}}(t) \leq \epsilon\right\}$; then $\mathbf{E} T \leq$ $(n e / 2 \delta)\left(x_{2}^{*}-x_{1}^{*}-\epsilon\right)+2+e$.

Compared to (7), we will see that the estimation of ET is much refined here. In [23], ET was estimated using Wald's equation. In Theorem 4, we estimate ET by directly calculating the expectation of some stopping times, and the upper bound decreases by $\left[\left(x_{2}^{*}-x_{1}^{*}\right)(2 / \delta-e / 2 \delta)+2+e \epsilon / 2 \delta\right] n-$ $2-e$. Since $\delta \leq \epsilon / 2 n$, a more detailed examination can obtain that the decrease is at least $n^{2}$, which is a much huge improvement, especially when the social group size $n$ is large.

In Theorem 4, the noise strength is still taken as $\delta \leq$ $\epsilon / 2 n$ as in Proposition 3. However, this does not mean the noise intervention fails to work when noise strength exceeds $\epsilon / 2 n$. Actually, the restriction of noise strength bound only facilitates the proof of Proposition 3, and simulations show that when noise strength exceeds $\epsilon / 2 n$, the disagreement can still be eliminated (see Section 4).

To prove Theorem 4, some lemmas are needed.

Lemma 5. Let $U_{0}=0$ and $U_{t}=\sum_{1}^{t} \xi(k), t \geq 1$, and define $T_{U}^{L}=\inf \left\{t \geq 0: U_{t} \geq L\right\}$ with $L>0$; then $\mathbf{E} T_{U}^{L} \leq(L / \delta+1)$.

Proof. Take arbitrary $\lambda \in[0, \delta]$, and let $T_{\lambda}=\inf \left\{t \geq 0: U_{t} \geq\right.$ $\lambda\}$; then it is easy to check that $T_{\lambda}$ is a stopping time. Note that $\{\xi(k), k \geq 1\}$ are independent and uniformly distributed on $[0, \delta]$, and let $f(y)$ be the probability density function of $\xi(k), k \geq 1$; then $f(y)=1 / \delta$ for $y \in[0, \delta]$, and hence

$$
\begin{aligned}
& P\left\{\max _{k \leq t} U_{k}<\lambda\right\}=P\left\{U_{t}<\lambda\right\}=\int_{0}^{\lambda} P\{\xi(2)+\cdots \\
& \left.+\xi(t)<\lambda-\lambda_{1}\right\} f\left(\lambda_{1}\right) d \lambda_{1}=\frac{1}{\delta} \\
& \quad \cdot \int_{0}^{\lambda} \int_{0}^{\lambda-\lambda_{1}} P\left\{\xi(3)+\cdots+\xi(t)<\lambda-\lambda_{1}-\lambda_{2}\right\} \\
& \cdot f\left(\lambda_{2}\right) d \lambda_{2} d \lambda_{1}=\cdots=\frac{1}{\delta^{t-2}} \\
& \quad \cdot \int_{0}^{\lambda} \int_{0}^{\lambda-\lambda_{1}} \cdots \int_{0}^{\lambda-\sum_{1}^{t-2} \lambda_{k}} P\left\{\xi(t)<\lambda-\sum_{1}^{t-1} \lambda_{k}\right\} \\
& \quad f\left(\lambda_{t-1}\right) d \lambda_{t-1} \cdots d \lambda_{1}=\frac{1}{\delta^{t}} \\
& \quad \cdot \int_{0}^{\lambda} \int_{0}^{\lambda-\lambda_{1}} \cdots \int_{0}^{\lambda-\sum_{1}^{t-2} \lambda_{k}}\left(\lambda-\sum_{1}^{t-1} \lambda_{k}\right) d \lambda_{t-1} \cdots d \lambda_{1} .
\end{aligned}
$$

$$
\begin{aligned}
P\left\{\max _{k \leq t} R_{k}<\lambda\right\} & =\int_{0}^{\lambda} P\left\{\xi(2)+\cdots+c \xi(t)<\lambda-\lambda_{1}\right\} f\left(\lambda_{1}\right) d \lambda_{1}=\frac{1}{\delta} \int_{0}^{\lambda} P\left\{\xi(2)+\cdots+c \xi(t)<\lambda-\lambda_{1}\right\} d \lambda_{1} \\
& =\frac{1}{\delta^{2}} \int_{0}^{\lambda} \int_{0}^{\lambda-\lambda_{1}} P\left\{\xi(3)+\cdots+c \xi(t)<\lambda-\lambda_{1}-\lambda_{2}\right\} d \lambda_{2} d \lambda_{1}=\cdots
\end{aligned}
$$

Suppose $L=m \delta+\lambda_{0}$, where $m$ is a nonnegative integer such that $\lambda_{0} \in[0, \delta)$. Define $T_{\delta}^{(0)}=0, T_{\delta}^{(1)}=T_{\delta}$, and $T_{\delta}^{(j+1)}=$ $\inf \left\{t \geq 1: U_{T_{\delta}^{j}+t}-U_{T_{\delta}^{j}} \geq \delta\right\}, j \geq 1$, where $T_{\delta}^{j}=\sum_{1}^{j} T_{\delta}^{(k)}$; then $\left\{T_{\delta}^{(j)}, j \geq 1\right\}$ are the copies of $T_{\delta}$, and hence i.i.d. with $T_{\delta}$. By (11), we have $\mathbf{E} T_{\delta}=e$; hence $\mathbf{E} T_{\delta}^{m}=m e$. Further, $T_{m \delta}^{\left(\lambda_{0}\right)}=$ $\inf \left\{t \geq 1: U_{T_{m \delta}+t}-U_{T_{m \delta}} \geq \lambda_{0}\right\}$ is $\sigma\left(\xi_{T_{m \delta}+1}, \ldots\right)$ measurable and hence independent with $\sigma\left(\xi\left(T_{m \delta}\right)\right)$. By (11), we have $\mathbf{E} T_{m \delta}^{\left(\lambda_{0}\right)}=$ $e^{\lambda_{0} / \delta}$. Since $\sum_{1}^{m}\left(U_{T_{\delta}^{k}}-U_{T_{\delta}^{k-1}}\right)+\left(U_{T_{U}^{L}}-U_{T_{\delta}^{m}}\right) \geq U_{T_{U}^{L}}$, we have $\mathbf{E} T_{U}^{L} \leq \mathbf{E}\left(\sum_{1}^{m} T_{\delta}^{(k)}+T_{m \delta}^{\left(\lambda_{0}\right)}\right)=m e+e^{\lambda_{0} / \delta}$. Since $m=\left(L-\lambda_{0}\right) / \delta$, $0 \leq \lambda_{0} / \delta<1$, the conclusion is obtained.

Lemma 6. Let $R_{1}=\xi(1), R_{t}=\sum_{1}^{t-1} \xi_{i}+c \xi(t), t \geq 2$ with $c>1$, and define $T_{\lambda}=\inf \left\{t \geq 1: R_{t} \geq \lambda\right\}$ for $\lambda \in(0, \delta]$; then $\mathbf{E} T_{\lambda} \leq(1 / c)\left(e^{\lambda / \delta}-1\right)$.

Proof. Given $\lambda \leq \delta$, we have 


$$
\begin{aligned}
& =\frac{1}{\delta^{t-1}} \int_{0}^{\lambda} \int_{0}^{\lambda-\lambda_{1}} \cdots \int_{0}^{\lambda-\lambda_{1}-\cdots-\lambda_{t-2}} P\left\{c \xi(t)<\lambda-\lambda_{1}-\cdots-\lambda_{t-1}\right\} d \lambda_{t-1} \cdots d \lambda_{1} \\
& =\frac{1}{c \delta^{t}} \int_{0}^{\lambda} \int_{0}^{\lambda-\lambda_{1}} \cdots \int_{0}^{\lambda-\lambda_{1}-\cdots-\lambda_{t-2}}\left(\lambda-\lambda_{1}-\cdots-\lambda_{t-1}\right) d \lambda_{t-1} \cdots d \lambda_{1} .
\end{aligned}
$$

Since

$$
\begin{gathered}
\int_{0}^{\lambda-\lambda_{1}-\cdots-\lambda_{t-2}}\left(\lambda-\lambda_{1}-\cdots-\lambda_{t-1}\right) d \lambda_{t-1} \\
=-\left.\frac{\left(\lambda-\lambda_{1}-\cdots-\lambda_{t-1}\right)^{2}}{2}\right|_{0} ^{\lambda-\lambda_{1}-\cdots-\lambda_{t-2}} \\
=\frac{\left(\lambda-\lambda_{1}-\cdots-\lambda_{t-2}\right)^{2}}{2}
\end{gathered}
$$

it has

$$
\begin{aligned}
& P\left\{\max _{k \leq t} R_{k}<\lambda\right\}=\frac{1}{c \delta^{t}} \\
& \cdot \int_{0}^{\lambda} \cdots \int_{0}^{\lambda-\lambda_{1}-\cdots-\lambda_{t-3}} \frac{\left(\lambda-\lambda_{1}-\cdots-\lambda_{t-2}\right)^{2}}{2} d \lambda_{t-2} \\
& \cdots d \lambda_{1}=\cdots=\frac{1}{c \delta^{t}} \int_{0}^{\lambda} \frac{\left(\lambda-\lambda_{1}\right)^{t-1}}{(t-1) !} d \lambda_{1}=\frac{1}{c \delta^{t}} \frac{\lambda^{t}}{t !} \\
& =\frac{1}{c} \frac{(\lambda / \delta)^{t}}{t !} .
\end{aligned}
$$

Thus

$$
\begin{aligned}
\mathbf{E} T_{\lambda} & =\sum_{t=1}^{\infty} P\left\{T_{\lambda} \geq t\right\}=\sum_{t=1}^{\infty} P\left\{\max _{k \leq t} R_{k}<\lambda\right\} \\
& =\frac{1}{c} \sum_{t=1}^{\infty} \frac{(\lambda / \delta)^{t}}{t !}=\frac{1}{c}\left(e^{\lambda / \delta}-1\right),
\end{aligned}
$$

and $\mathbf{E} T_{\delta}=(1 / c)(e-1)$ especially.

Proof of Theorem 4. It is easy to know by the proof of Proposition 3 that, when $t<T$, the group $\mathscr{V}_{1}$ is separated from $\mathscr{V}_{2}$ (refer to the proof of Theorem 3 of [24]); then, for $t<T$,

$$
\begin{aligned}
x_{1}(t)= & \frac{1}{\left|\mathscr{V}_{1}\right|} \sum_{j \in \mathscr{V}_{1}} x_{j}(t-1)+\xi(t) \\
= & \frac{1}{\left|\mathscr{V}_{1}\right|}\left(\sum_{j \in \mathscr{V}_{1}} \frac{1}{\left|\mathscr{V}_{1}\right|} \sum_{k \in \mathscr{V}_{1}} x_{k}(t-2)+\xi(t-1)\right) \\
& +\xi(t)
\end{aligned}
$$

$$
\begin{aligned}
& =\frac{1}{\left|\mathscr{V}_{1}\right|} \sum_{j \in \mathscr{V}_{1}} x_{j}(t-2)+\frac{1}{\left|\mathscr{V}_{1}\right|} \xi(t-1)+\xi(t) \\
& =\frac{1}{\left|\mathscr{V}_{1}\right|} \sum_{j \in \mathscr{V}_{1}} x_{j}(0)+\sum_{k=1}^{t-1} \frac{1}{\left|\mathscr{V}_{1}\right|} \xi(k)+\xi(t) \\
& =x_{1}^{*}+\frac{1}{\left|\mathscr{V}_{1}\right|} \sum_{k=1}^{t-1} \xi(k)+\xi(t) .
\end{aligned}
$$

Let $R_{1}=\xi(1) ; R_{t}=\sum_{k=1}^{t-1} \xi(k)+\left|\mathscr{V}_{1}\right| \xi(t), t \geq 2$. Since the system reaches $\delta$-consensus at $T+1$, while at $T$ it has $x_{2}^{*}-$ $x_{1}(T) \leq \epsilon$, then by $(16)$

$$
T=\inf \left\{t>0: R_{t} \geq\left|\mathscr{V}_{1}\right|\left(x_{2}^{*}-x_{1}^{*}-\epsilon\right)\right\}
$$

Denote $l=x_{2}^{*}-x_{1}^{*}-\epsilon$, and let $U_{0}=0, U_{t}=\sum_{k=1}^{t} \xi(k), t>0$. For $l \geq \delta /\left|\mathscr{V}_{1}\right|$

$$
\begin{aligned}
P\left\{R_{t}<\left|\mathscr{V}_{1}\right| l\right\} & =\int_{0}^{\delta} P\left\{U_{t-1}<\left|\mathscr{V}_{1}\right|(l-\lambda)\right\} f(\lambda) d \lambda \\
& =\frac{1}{\delta} \int_{0}^{\delta} P\left\{U_{t-1}<\left|\mathscr{V}_{1}\right|(l-\lambda)\right\} d \lambda .
\end{aligned}
$$

If $\delta /\left|\mathscr{V}_{1}\right| \leq l \leq \delta$, by Lemma 5 and (18),

$$
\begin{aligned}
\mathbf{E} T & =\sum_{t=1}^{\infty} P\{T \geq t\}=1+\sum_{t=2}^{\infty} P\left\{R_{t}<\left|\mathscr{V}_{1}\right| l\right\} \\
& =1+\frac{1}{\delta} \int_{0}^{l}\left(1+\sum_{t=1}^{\infty} P\left\{U_{t}<\left|\mathscr{V}_{1}\right|(l-\lambda)\right\}\right) d \lambda \\
& \leq 2+\frac{1}{\delta} \int_{0}^{l}\left(\frac{\left|\mathscr{V}_{1}\right|(l-\lambda)}{\delta}+1\right) e d \lambda \\
& =\frac{\left|\mathscr{V}_{1}\right| e}{2}\left(\frac{l}{\delta}\right)^{2}+2+e .
\end{aligned}
$$

Otherwise if $l>\delta$, by Lemma 5 and (18),

$$
\begin{aligned}
\mathbf{E} T & =\sum_{t=1}^{\infty} P\{T \geq t\}=1+\sum_{t=1}^{\infty} P\left\{R_{t}<\left|\mathscr{V}_{1}\right| l\right\} \\
& =1+\frac{1}{\delta} \int_{0}^{\delta}\left(1+\sum_{t=1}^{\infty} P\left\{U_{t}<\left|\mathscr{V}_{1}\right|(l-\lambda)\right\}\right) d \lambda
\end{aligned}
$$




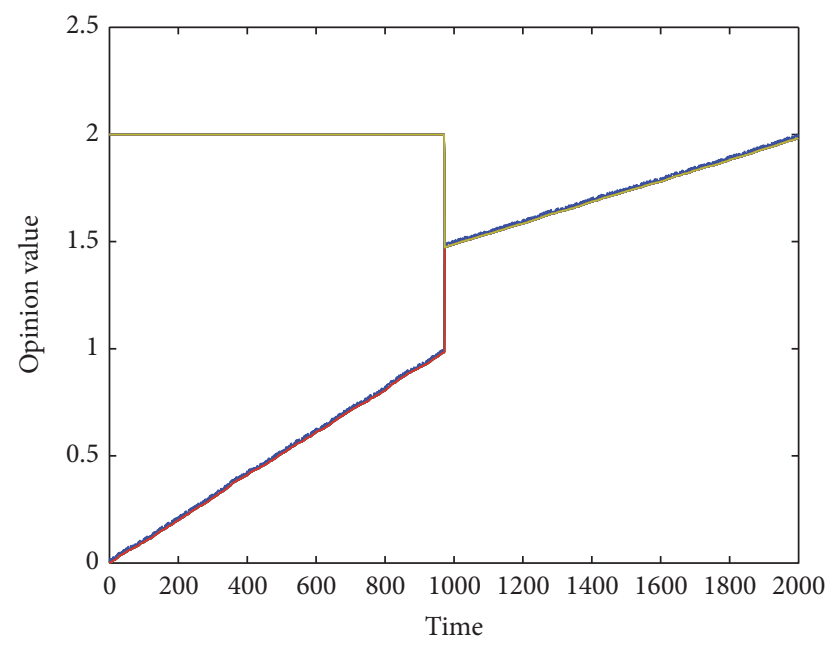

Figure 1: Opinion evolution of system (2)-(5) of 20 agents with noise uniformly distributed on $[0,0.02] \epsilon$. The initial opinion value $x(0)=\left[\begin{array}{llllllllllllllllllll}0 & 0 & 0 & 0 & 0 & 0 & 0 & 0 & 0 & 0 & 2 & 2 & 2 & 2 & 2 & 2 & 2 & 2 & 2 & 2\end{array}\right]$, confidence threshold $\epsilon=1$, and noise strength $\delta=0.02$.

$$
\begin{aligned}
& \leq 2+\frac{1}{\delta} \int_{0}^{\delta}\left(\frac{\left|\mathscr{V}_{1}\right|(l-\lambda)}{\delta}+1\right) e d \lambda \\
& =\left|\mathscr{V}_{1}\right|\left(\frac{l}{\delta}-\frac{1}{2}\right) e+2+e .
\end{aligned}
$$

Since $\left|\mathscr{V}_{1}\right| \leq n / 2$, (19) and (20) imply

$$
\mathbf{E} T \leq \frac{n e}{2 \delta} l+2+e .
$$

For $l \in\left(0, \delta /\left|\mathscr{V}_{1}\right|\right)$, by Lemma 6 , we have

$$
\begin{aligned}
\mathbf{E} T & =\sum_{t=1}^{\infty} P\{T \geq t\}=1+\sum_{t=1}^{\infty} P\left\{R_{t}<\left|\mathscr{V}_{1}\right| l\right\} \\
& =\frac{1}{\left|\mathscr{V}_{1}\right|}\left(e^{\left|\mathscr{V}_{1}\right| l / \delta}-1\right)+1 .
\end{aligned}
$$

Since $\left|\mathscr{V}_{1}\right| l / \delta \leq 1$ and $e^{x}=1+x+o\left(x^{2}\right)$ for $x \in(0,1)$, by (22), it has $\mathbf{E} T \leq 2 l / \delta$, implying the conclusion together with (21).

\section{Simulations}

In this part, we will present some simulation results to show the how the opinions reach consensus under the driven of noise. Take $n=20, \epsilon=1$. According to Proposition 3, take noise strength $\delta=0.02 \epsilon<0.025 \epsilon$; then Figure 1 shows that the divisive opinions reach consensus under the drive of noise. The time scale here obviously decreases compared to that in [24]. Second, we take the noise strength $\delta=$ $0.04 \epsilon$, which exceeds the upper bound $0.025 \epsilon$; then Figure 2 shows that the disagreement can also be eliminated, and the consensus time gets interestingly shorter. In addition, though we only analyze the divisive systems with only two subgroups,

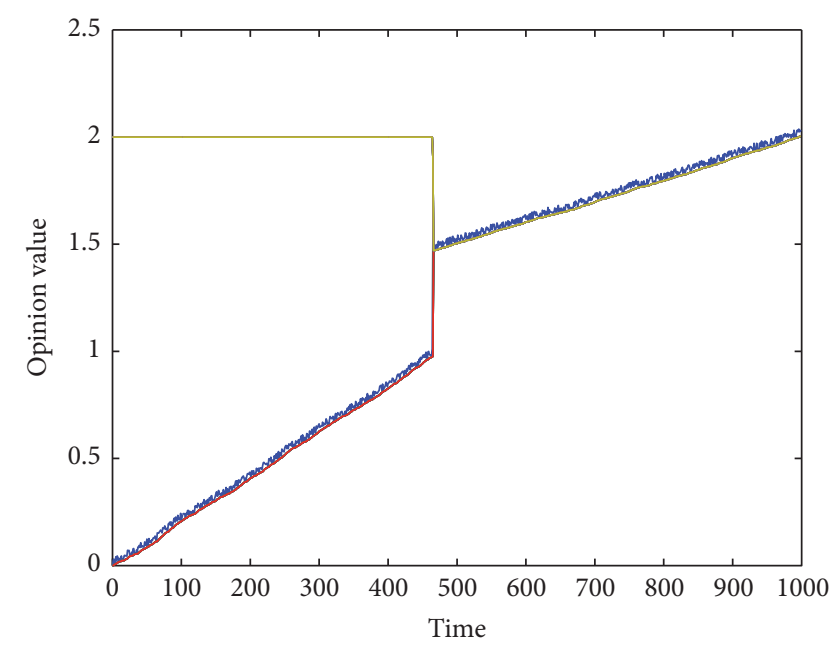

Figure 2: Opinion evolution of system (2)-(5) of 20 agents with noise uniformly distributed on $[0,0.04] \epsilon$. The initial opinion value $x(0)=\left[\begin{array}{llllllllllllllllllll}0 & 0 & 0 & 0 & 0 & 0 & 0 & 0 & 0 & 0 & 2 & 2 & 2 & 2 & 2 & 2 & 2 & 2 & 2 & 2\end{array}\right]$, confidence threshold $\epsilon=1$, and noise strength $\delta=0.04$.

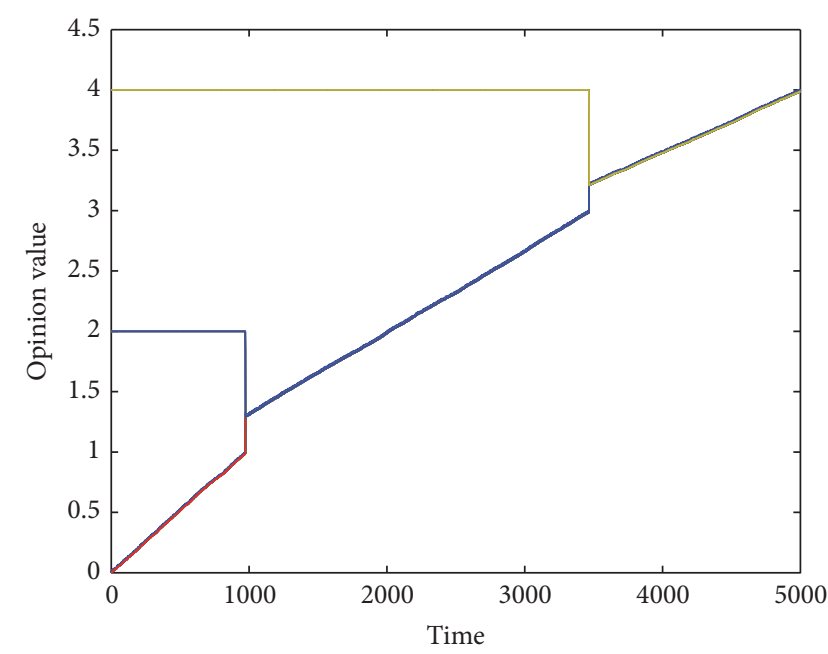

Figure 3: Opinion evolution of system (2)-(5) of 20 agents with noise uniformly distributed on $[0,0.02] \epsilon$. The initial opinion value $x(0)=\left[\begin{array}{llllllllllllllllllll}0 & 0 & 0 & 0 & 0 & 0 & 0 & 0 & 0 & 0 & 2 & 2 & 2 & 2 & 2 & 4 & 4 & 4 & 4 & 4\end{array}\right]$, confidence threshold $\epsilon=1$, and noise strength $\delta=0.02$.

we provide simulation result of three subgroups to further show how the separated subgroups get seriatim merged (see Figure 3).

\section{Conclusions}

In this paper, we mainly aimed at analyzing the finite stopping time when the disagreement was eliminated by a simple noise intervention strategy. Compared to the results obtained in the previous studies, our result in this paper largely gets refined. The analysis method used in this paper would provide another tool for subtly investigating the stopping time when the system gets consensus and is expected to get further developed in the near future. 


\section{Disclosure}

Yong Ding and Lipo Mo are co-first authors.

\section{Conflicts of Interest}

The authors declare that there are no conflicts of interest regarding the publication of this paper.

\section{Authors' Contributions}

Yong Ding and Lipo Mo contributed equally to this work.

\section{Acknowledgments}

This work is supported by the National Natural Science Foundation (NNSF) of China (Grant no. 61304155) and the Beijing Municipal Government Foundation for Talents (Grant no. 2012D005003000005).

\section{References}

[1] C. Castellano, S. Fortunato, and V. Loreto, "Statistical physics of social dynamics," Reviews of Modern Physics, vol. 81, no. 2, pp. 591-646, 2009.

[2] A. V. Proskurnikov and R. Tempo, "A tutorial on modeling and analysis of dynamic social networks. Part I," Annual Reviews in Control, vol. 43, pp. 65-79, 2017.

[3] P. Jia, A. MirTabatabaei, N. E. Friedkin, and F. Bullo, "Opinion dynamics and the evolution of social power in influence networks," SIAM Review, vol. 57, no. 3, pp. 367-397, 2015.

[4] M. H. DeGroot, "Reaching a consensus," Journal of the American Statistical Association, vol. 69, no. 345, pp. 118-121, 1974.

[5] N. E. Friedkin and E. C. Johnsen, "Social influence networks and opinion change," Advances in Group Processes, vol. 16, pp. 1-29, 1999.

[6] G. Deffuant, D. Neau, F. Amblard, and G. Weisbuch, "Mixing beliefs among interacting agents," Advances in Complex Systems (ACS), vol. 3, pp. 87-98, 2000.

[7] U. Krause, "A discrete nonlinear and non-autonomous model of consensus formation," in Communications in Difference Equations (Poznan, 1998), S. Elaydi, G. Ldas, J. Popenda, and J. Rakowski, Eds., pp. 227-236, Gordon and Breach, Amsterdam, 2000.

[8] R. Hegselmann and U. Krause, "Opinion dynamics and bounded confidence: models, analysis and simulation," Journal of Artificial Societies and Social Simulation, vol. 5, no. 3, 2002.

[9] V. D. Blondel, J. M. Hendrickx, and J. N. Tsitsiklis, “On Krause’s multi-agent consensus model with state-dependent connectivity," Institute of Electrical and Electronics Engineers Transactions on Automatic Control, vol. 54, no. 11, pp. 2586-2597, 2009.

[10] S. Fortunato, "The Krause-Hegselmann consensus model with discrete opinions," International Journal of Modern Physics C, vol. 15, article 1021, 2004.

[11] G. Toscani, "Kinetic models of opinion formation," Communications in Mathematical Sciences, vol. 4, no. 3, pp. 481-496, 2006.

[12] S. Parongama and B. K. Chakrabarti, Sociophysics: An Introduction, Oxiford University Press, Oxford, UK, 2013.

[13] S. Galam, Sociophysics, Springer, New York, NY, USA, 2012.
[14] M. Mäs, A. Flache, and D. Helbing, "Individualization as driving force of clustering phenomena in humans," PLoS Computational Biology, vol. 6, no. 10, Article ID 1000959, 2010.

[15] S. Grauwin and P. Jensen, "Opinion group formation and dynamics: Structures that last from nonlasting entities," Physical Review E: Statistical, Nonlinear, and Soft Matter Physics, vol. 85, no. 6, Article ID 066113, 2012.

[16] A. Carro, R. Toral, and M. San Miguel, "The role of noise and initial conditions in the asymptotic solution of a bounded confidence, continuous-opinion model," Journal of Statistical Physics, vol. 151, no. 1-2, pp. 131-149, 2013.

[17] M. Pineda, R. Toral, and E. Hernández-García, "The noisy Hegselmann-Krause model for opinion dynamics," The European Physical Journal B, vol. 86, article 490, 2013.

[18] W. Horsthemke and R. Lefever, Noise-Induced Transitions, vol. 15 of Springer Series in Synergetics, Springer, Berlin, Germany, 1984.

[19] J. García-Ojalvo and J. M. Sancho, Noise in Spatially Extended Systems, Institute for Nonlinear Science, Springer-Verlag, New York, NY, USA, 1999.

[20] L. Q. Zhou, X. Jia, and Q. Ouyang, "Experimental and numerical studies of noise-induced coherent patterns in a subexcitable system," Physical Review Letters, vol. 88, Article ID 138301, 2002.

[21] R. Müller, K. Lippert, A. Kühnel, and U. Behn, "First-order nonequilibrium phase transition in a spatially extended system," Physical Review E: Statistical, Nonlinear, and Soft Matter Physics, vol. 56, no. 3, pp. 2658-2662, 1997.

[22] M. G. Clerc, C. Falcon, and E. Tirapegui, "Additive noise induces front propagation," Physical Review Letters, vol. 94, no. 14, Article ID 148302, 2005.

[23] W. Su, G. Chen, and Y. Hong, "Noise leads to quasi-consensus of Hegselmann-Krause opinion dynamics," Automatica, vol. 85, pp. 448-454, 2017.

[24] W. Su, G. Chen, and Y. Yu, "Finite-time elinimation of disagreement of opinion dynamics via covert noise," https:/arxiv.org/ abs/1611.01732, 2016. 


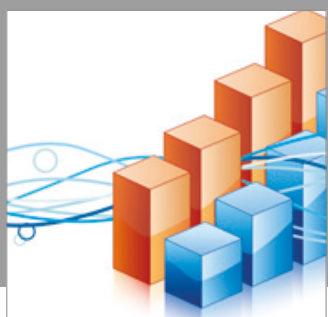

Advances in

Operations Research

vatersals

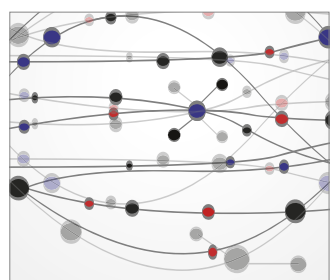

\section{The Scientific} World Journal
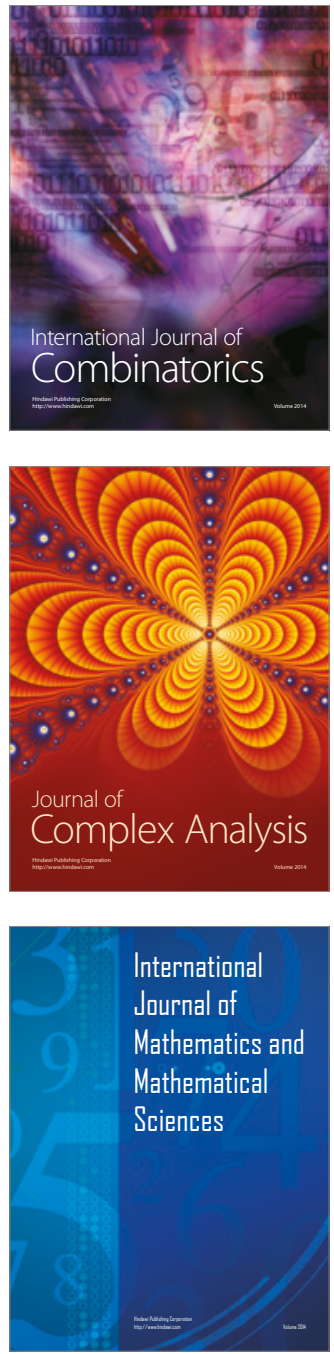
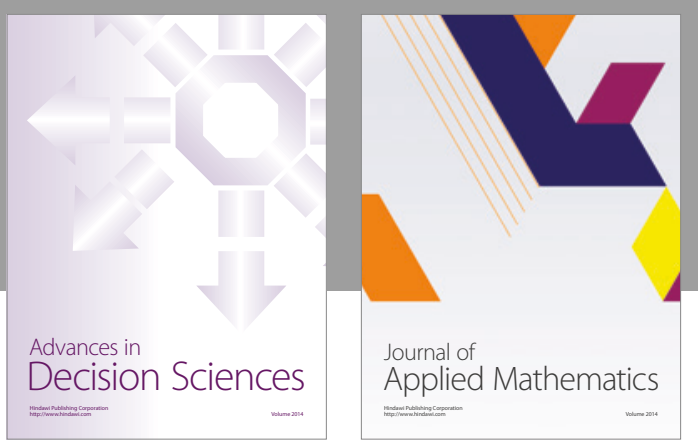

Algebra

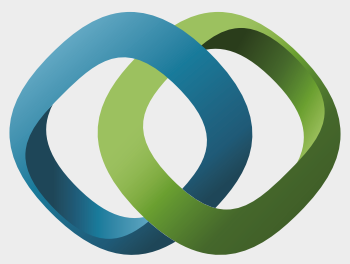

\section{Hindawi}

Submit your manuscripts at

https://www.hindawi.com
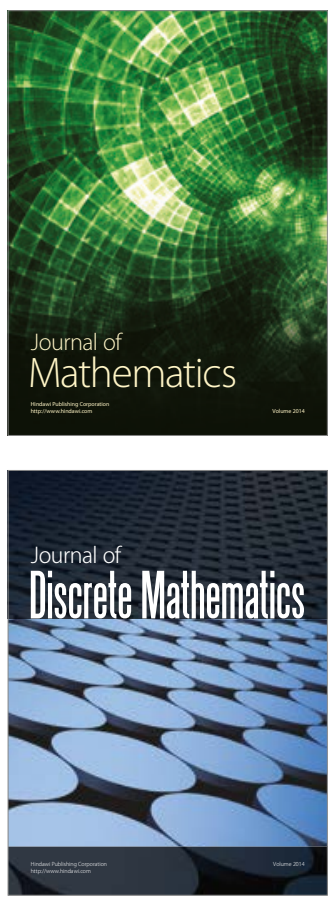

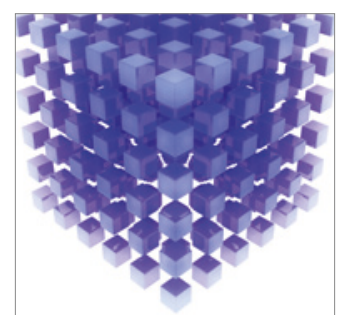

Mathematical Problems in Engineering
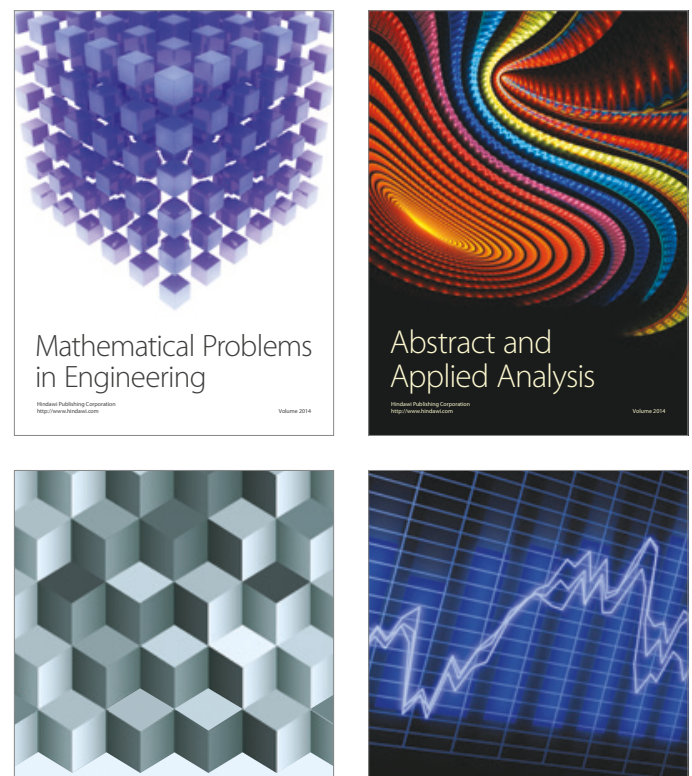

Journal of

Function Spaces

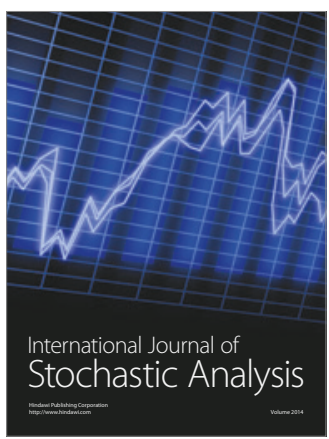

Probability and Statistics
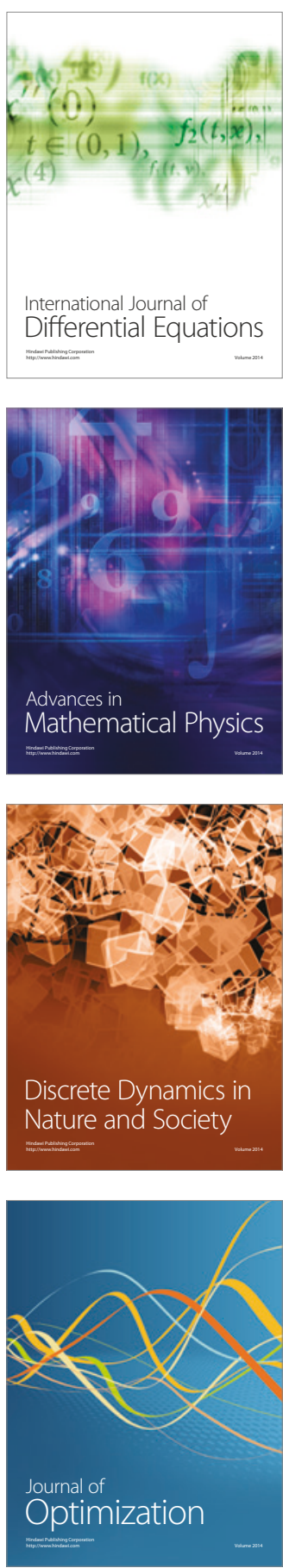